Proceedings of the Edinburgh Mathematical Society (2004) 47, 101-109 (C)

DOI:10.1017/S0013091502001062 Printed in the United Kingdom

\title{
THE EMBEDDING OF A CYCLIC PERMUTABLE SUBGROUP IN A FINITE GROUP. II
}

\author{
J. COSSEY ${ }^{1}$ AND S. E. STONEHEWER ${ }^{2}$ \\ ${ }^{1}$ Department of Mathematics, Australian National University, \\ Canberra, ACT 0200, Australia (john.cossey@maths.anu.edu.au) \\ ${ }^{2}$ Mathematics Institute, University of Warwick, \\ Coventry CV4 7AL, UK (Stewart@stonehewer.freeserve.co.uk)
}

(Received 22 November 2002)

\begin{abstract}
In two previous papers we established the structure of the normal closure of a cyclic permutable subgroup $A$ of a finite group, first when $A$ has odd order and second when $A$ has even order, but with an extra hypothesis that was unnecessary in the odd case. Here we describe the most general situation without any restrictions on $A$.
\end{abstract}

Keywords: cyclic subgroup; permutable subgroup; power automorphism

2000 Mathematics subject classification: Primary 20D35; 20D40

\section{Introduction and statement of results}

Let $A$ be a subgroup of a group $G$. Then $A$ is said to be permutable (or quasinormal) if $A X=X A$ for all subgroups $X$ of $G$. Thus $A$ is permutable precisely when the product $A X$ is a subgroup for each subgroup $X$ of $G$. Suppose that

$G$ is finite and $A$ is a cyclic permutable subgroup.

When $A$ has odd order, then we showed in $[\mathbf{3}]$ that $[A, G]$ is abelian and $A$ acts on it by conjugation as a group of power automorphisms. When $A$ has even order, this result is not true in general. But we showed in [4] that $N=[A, G]^{\prime}$ has order at most 2 and lies in $A$, and $A$ acts by conjugation on $[A, G] / N$ as a group of power automorphisms, provided in each product $A X$, with $X$ cyclic, the Sylow 2-subgroup has a modular subgroup lattice. (For odd primes $p$, the Sylow $p$-subgroups of $A X$ always have a modular subgroup lattice (see $\left[6\right.$, Hauptsatz I]).) In [4] we also gave examples in which $[A, G]^{\prime}$ has order 2 . In this paper we assume no additional hypothesis about $A$ and $G$. It turns out that $[A, G]$ is always nilpotent of class at most 2 . Also, if we denote by $2^{\prime}$ the set of odd primes, then the $2^{\prime}$-component of $[A, G]$ is abelian and $A$ acts on it as a group of power automorphisms. If $A_{2}$ is the 2-component of $A$, then $\left[A_{2}, G\right]$ is the 2-component of $[A, G]$. We find that $\left[A_{2}, G, A\right]$ is a normal subgroup of $G$ lying in the centre of $[A, G]$ and $A$ acts on $\left[A_{2}, G, A\right]$ as a group of power automorphisms. 
We denote by $A_{2^{\prime}}$ the $2^{\prime}$-component of $A$ (always a cyclic group). Also we write $Z(G)$ for the centre of a group $G$. Then we shall prove the following theorem.

Theorem 1.1. Let $A$ be a cyclic permutable subgroup of a finite group $G$. Then

(i) $[A, G]=\left[A_{2}, G\right] \times\left[A_{2^{\prime}}, G\right]$;

(ii) $\left[A_{2^{\prime}}, G\right]$ is an abelian $2^{\prime}$-group;

(iii) $A$ acts by conjugation on $\left[A_{2^{\prime}}, G\right]$ as a group of power automorphisms;

(iv) $\left[A_{2}, G\right]$ is a 2-group of class at most 2;

(v) $\left[A_{2}, G, A\right]$ is a normal subgroup of $G$ and lies in $Z\left(\left[A_{2}, G\right]\right)$; and

(vi) $A$ acts by conjugation on $\left[A_{2}, G, A\right]$ as a group of power automorphisms.

All our arguments reduce quickly to the case where $G$ is a 2-group. Here our main results are the following.

Theorem 1.2. Let $A=\langle a\rangle$ be a cyclic permutable subgroup of a finite 2-group $G$. Then

(i) $[A, G, A] \triangleleft G$; and

(ii) $[A, G, A]=\{[u, a] \mid u \in[A, G]\}$.

Theorem 1.3. Let $A$ be a cyclic permutable subgroup of a finite 2-group $G$ and put $B=[A, G, A]$. Then

(i) $B \leqslant Z([A, G])$;

(ii) A centralizes $[A, G] / B$;

(iii) $A$ acts by conjugation on $B$ as a group of power automorphisms; and

(iv) $[A, G]$ has nilpotency class at most 2.

Remark 1.4. It was shown by Cooper in $[2]$ that a power automorphism of a finite abelian group is always universal, i.e. each element maps to the same power.

In $\S 2$ we deduce Theorems 1.1 and 1.3 from Theorem 1.2. Then we establish several preliminary lemmas for the proof of Theorem 1.2 in $\S \S 3$ and 4 . We denote by $H^{G}$ and $H_{G}$, respectively, the normal closure and the core of a subgroup $H$ in a group $G$. When $G$ is a $p$-group, for some prime $p$, then $\Omega(G)$ is the subgroup generated by the elements of order $p$. All other notation is standard. 


\section{Preliminary lemmas and proofs of Theorems 1.1 and 1.3}

The proof of Theorem 1.3 will follow easily from Theorem 1.2 and one of the most useful results in $[4]$, which we record again here for convenience.

Lemma 2.1 (see Lemma 2.3 in [4]). Let $G=A X$ be a finite 2-group, where $A=\langle a\rangle$ and $X=\langle x\rangle$ are cyclic subgroups and $A$ is permutable in $G$. Then

(i) $G$ is metacyclic;

(ii) $G^{\prime}=\langle[a, x]\rangle$;

(iii) for each integer $i,\left\langle\left[a^{i}, x\right]\right\rangle=\left\langle[a, x]^{i}\right\rangle$;

(iv) a conjugates $[a, x]$ to a power congruent to 1 modulo 4; and

(v) each element of $G^{\prime}$ has the form $\left[a^{i}, x\right]$, for some integer $i$.

From part (iii) we immediately obtain the following corollary.

Corollary 2.2. Let $A=\langle a\rangle$ be a cyclic permutable subgroup of a finite 2-group $G$. Then $[A, G]=\langle[a, g] \mid g \in G\rangle$.

Proof of Theorem 1.3. We have a cyclic permutable subgroup $A=\langle a\rangle$ of a finite 2-group $G$ and $B=[A, G, A]$. By Theorem 1.2 (ii), each cyclic subgroup of $B$ has the form $\langle[u, a]\rangle=K$, say, for some element $u$ in $[A, G]$. But by Lemma 2.1 (i), $A$ normalizes $K$ and so $A$ normalizes every subgroup of $B$. By Theorem 1.2 (i), $B \triangleleft G$ and therefore it follows that $A^{G}$ normalizes every subgroup of $B$. Thus $B\left(\leqslant A^{G}\right)$ has all its subgroups normal and by $[\mathbf{5}]$ and $[\mathbf{1}]$ (see also $[\mathbf{9}$, Theorem 2.3.12]), $B$ is either abelian or the direct product of a quaternion group of order 8 and an elementary abelian 2-group. In the latter case, $B$ has exponent 4 and so $A$ centralizes $B$, by Lemma 2.1 (iv). But then $A^{G}$ also centralizes $B$ and $B$ is abelian, a contradiction. Thus $B$ must be abelian and then (iii) follows from $[\mathbf{2}]$.

Suppose that $a$ conjugates each element of $B$ to its $r$ th power. Let $x \in B$ and $g \in G$. Then

$$
x^{[a, g]}=x^{a^{-1} g^{-1} a g}=\left(\left(x^{a^{-1} g^{-1}}\right)^{r}\right)^{g}=\left(x^{r}\right)^{a^{-1}}=x,
$$

and so from Corollary 2.2 we see that $[A, G]$ centralizes $B$. Therefore, (i) is true. Part (ii) is trivial. Finally, because $B \triangleleft G$, we deduce that $A^{G}$ centralizes $[A, G] / B$ and thus $[A, G] / B$ is abelian. Hence (iv) is true.

In order to prove Theorem 1.1, all we need now is a well-known basic result.

Lemma 2.3 (see Lemma 5.2.11 in [9]). Let $A$ be a cyclic permutable subgroup of a group $G$. Then every subgroup of $A$ is also permutable in $G$.

Proof of Theorem 1.1. We have a cyclic permutable subgroup $A$ of a finite group $G$. By $[7], A$ is subnormal in $G$ and so $A^{G}=A[A, G]$ is nilpotent. Thus (i) follows immediately from $A=A_{2} \times A_{2^{\prime}}$. Also $\left[A_{2}, G\right]$ and $\left[A_{2^{\prime}}, G\right]$ are the 2- and $2^{\prime}$-components, respectively, of $[A, G]$. 
By Lemma 2.3, $A_{2}$ and $A_{2^{\prime}}$ are both permutable subgroups of $G$. Then parts (ii) and (iii) are proved in Theorem 1.1 of [3], bearing in mind that $A_{2}$ centralizes $\left[A_{2^{\prime}}, G\right]$.

Elements in $G$ of odd order normalize and therefore centralize $A_{2}$. Thus for any Sylow 2-subgroup $G_{2}$ of $G$, we have $A_{2} \leqslant G_{2}$ and $\left[A_{2}, G\right]=\left[A_{2}, G_{2}\right]$. Then (iv) follows from Theorem 1.3 (iv). Similarly, $\left[A_{2}, G, A\right]=\left[A_{2}, G_{2}, A_{2}\right]=B$, say. By Theorem 1.2, $B \triangleleft G_{2}$; and since the elements of odd order in $G$ actually centralize $\left[A_{2}, G\right]$, we even have $B \triangleleft G$. By Theorem $1.3(\mathrm{i}), B \leqslant Z\left(\left[A_{2}, G\right]\right)$. This proves (v).

Finally, (vi) follows from Theorem 1.3 (iii), observing that $A_{2^{\prime}}$ centralizes $B$.

In the remainder of this section we establish some new and record some old results that will be required for the proof of Theorem 1.2 in $\S \S 3$ and 4 . The first comes from [4] and is both applicable to, and important in, this more general situation.

Lemma 2.4. Let $A$ be a cyclic permutable subgroup of a finite 2-group $G$. Then $\left[A^{2}, G\right]=[A, G]^{2}$.

This allows us to prove our next result.

Lemma 2.5. Let $A=\langle a\rangle$ be a cyclic permutable subgroup of a finite 2-group $G=$ $\left\langle a, g_{1}, \ldots, g_{r}\right\rangle$. Then there is a commutator $\left[a, g_{i}\right]$ of order equal to the exponent of $[A, G]$.

Proof. We argue by induction on $|A|$. Let $[A, G]$ have exponent $2^{n}$ and let $2^{m}$ be the maximal order of a commutator $\left[a, g_{i}\right], 1 \leqslant i \leqslant r$. So $m \leqslant n$ and we may assume that $m \geqslant 1$, since $[A, G]=\left\langle\left[a, g_{i}\right] \mid i=1, \ldots, r\right\rangle^{G}$. By Lemma $2.3, A^{2}$ is permutable in $G$. Put $H=\left\langle a^{2}, g_{1}, \ldots, g_{r}\right\rangle$. Then $G=A H$. Also by induction there is a commutator $\left[a^{2}, g_{i}\right]$ of order equal to the exponent of $\left[A^{2}, H\right]$. But, by Lemma 2.4, $\left[A^{2}, H\right]=\left[A^{2}, G\right]=[A, G]^{2}$. Therefore, by Lemma 2.1 (iii) this exponent is $2^{m-1}$. But clearly it is either $2^{n}$ or $2^{n-1}$. Thus it must be $2^{n-1}$ and $m=n$ as required.

We recall that permutable subgroups of order 2 are well understood.

Lemma 2.6 (see Theorem 5.2.9 in [9]). Let $G$ be a finite 2-group and let $A$ be a permutable subgroup of order 2 . Then $[A, G] \leqslant Z(G)$.

In the proof of Theorem $1.2(\mathrm{i})$ we shall reduce to the case where $G$ is generated by $A$ and at most two other elements. The remaining results in this section examine that situation. They all extend trivially to the case where $G$ has any number of generators. The first has already appeared in [4].

Lemma 2.7 (see Lemma 3.3 in [4]). Let $A=\langle a\rangle$ be a cyclic permutable subgroup of a finite 2 -group $G=\langle a, x, y\rangle$. Then

$$
[A, G]=(A \cap[A, G])\langle[a, x]\rangle\langle[a, y]\rangle .
$$

Corollary 2.8. With the same hypotheses as Lemma 2.7 and for any integer $i \geqslant 0$, we have

$$
\left[A^{2^{i}}, G\right]=\left(A^{2^{i}} \cap\left[A^{2^{i}}, G\right]\right)\left\langle\left[a^{2^{i}}, x\right]\right\rangle\left\langle\left[a^{2^{i}}, y\right]\right\rangle .
$$


The proof is straightforward and may be omitted. A complementary result is the following.

Lemma 2.9. With the same hypotheses as Lemma 2.7 and for any integer $i \geqslant 0$, we have

$$
\left[A^{2^{i}}, G\right]=\left\langle\left[a^{2^{i}}, x\right]\right\rangle\left\langle\left[a^{2^{i}}, y\right]\right\rangle\left[A^{2^{i}}, G, G\right]
$$

Proof. We keep the same notation as in Corollary 2.8 and denote the right-hand side of (2.3) by $K$. Clearly, $[B, G] \geqslant K$. Conversely, $K \triangleleft G$ and, modulo $K, b$ commutes with $a, x$ and $y$, i.e. with $G$. Therefore, $[B, G] \leqslant K$ and so we have equality.

We need one more preliminary result for the proof of Theorem 1.2.

Lemma 2.10. With the same hypotheses as Lemma 2.7 and for any integer $i \geqslant 0$,

$$
\left[A^{2^{i}}, G, G\right] \leqslant\left\langle\left[a^{2^{i}}, x, y\right]\right\rangle\left\langle\left[a^{2^{i}}, y, x\right]\right\rangle\left[A^{2^{i+1}}, G\right] .
$$

Proof. Again we keep the notation of Corollary 2.8 and denote the right-hand side of (2.4) by $L$. By Lemmas 2.3 and 2.6 we have $[B, G, G, G] \leqslant\left[B^{2}\left[B^{2}, G\right], G\right]=\left[B^{2}, G\right]$. Therefore, $[B, G, G]$ is central in $G$ modulo $\left[B^{2}, G\right]$. Thus $L \triangleleft G$. But, by Lemma 2.1,

$$
[b, x, x] \in\left\langle[b, x]^{2}\right\rangle=\left\langle\left[b^{2}, x\right]\right\rangle \leqslant\left[B^{2}, G\right] .
$$

Similarly, $[b, y, y] \in\left[B^{2}, G\right]$. Since $A \cap[B, G] \leqslant B^{2}$, it follows that, modulo $L$, each of the three factors on the right-hand side of $(2.2)$ is centralized by $G$. Therefore, $[B, G, G] \leqslant L$ by Corollary 2.8, as required.

\section{Proof of Theorem 1.2 (i)}

We have a finite 2-group $G$ with a permutable cyclic subgroup $A=\langle a\rangle$ and we must show that $[A, G, A]=K$, say, is normal in $G$. We begin by reducing to the case where

$G$ is generated by $A$ and at most two other elements.

By Corollary 2.2, $[A, G]=\langle[a, g] \mid g \in G\rangle$. So a typical element of $[A, G]$ has the form $u=u_{1} u_{2} \ldots u_{m}$, where $u_{i}=\left[a, g_{i}\right], g_{i} \in G$. Then $K$ is generated by elements $v=[u, a]$, by Lemma 2.1 (iii). It is sufficient to show that $v^{y} \in K$ for all such $v$ and $y \in G$. But

$$
v^{y}=\left[u_{1} \ldots u_{m}, a\right]^{y}=\left[u_{1}, a\right]^{y_{1}}\left[u_{2}, a\right]^{y_{2}} \ldots\left[u_{m}, a\right]^{y_{m}},
$$

where $y_{i}=u_{i+1} u_{i+2} \ldots u_{m} y$. Thus with $G_{i}=\left\langle a, g_{i}, y_{i}\right\rangle$, we have

$$
\left[u_{i}, a^{y_{i}}\right] \in\left[A, G_{i}, A\right]^{y_{i}}=\left[A, G_{i}, A\right]
$$

if the theorem is true for $A$ as a permutable subgroup of $G_{i}$. Then each factor on the right-hand side of (3.2) lies in $K$ and the theorem follows. Therefore, we may assume that (3.1) holds and so

$$
G=\langle a, x, y\rangle
$$

Suppose that the theorem is false when (3.1) holds and let $G$ (given by (3.3)) be a counterexample of minimal order. Then $K_{G}=1$ and $K \neq 1$. We distinguish two cases. 
Case 1. Suppose that $\langle[a, x]\rangle \cap\langle[a, y]\rangle \neq 1$. By Lemma 2.7 we may assume without loss of generality that $[a, x, a] \neq 1$. Then $1 \neq\langle[a, x, a]\rangle \leqslant\langle[a, x]\rangle$ by Lemma 2.1 (ii). So $N=\Omega(\langle[a, x]\rangle)=\Omega(\langle[a, y]\rangle) \leqslant K \cap Z(G)$, contradicting $K_{G}=1$.

Case 2. Suppose that $\langle[a, x]\rangle \cap\langle[a, y]\rangle=1$. Without loss of generality we may assume that $|[a, x]| \geqslant|[a, y]|$. So $|[a, x]|=2^{n}$ (say) is the exponent of $[A, G]$, by Lemma 2.5. If $|[a, y]|<2^{n}$, then $|[a, x y]|=2^{n}$, since $G=\langle a, x y, y\rangle$ (using Lemma 2.5 again). Therefore, replacing $y$ by $x y$ if necessary, we may assume that

$$
|[a, x]|=|[a, y]|=2^{n} \text { is the exponent of }[A, G] .
$$

To simplify notation, we write $A_{i}=A^{2^{i}}$ and $a_{i}=a^{2^{i}}$ for each integer $i \geqslant 0$. Choose $i$ such that $a$ acts non-trivially on $\left[A_{i}, G\right]$ and trivially on $\left[A_{i+1}, G\right]$. So $0 \leqslant i<n-2$, by Lemma 2.1 (iv). (We could have chosen our counterexample $G$ with $|A|$ minimal. Then $\left[A^{2}, G, A^{2}\right] \triangleleft G$ and so $\left[A^{2}, G, A^{2}\right]=1$. This implies that $a$ centralizes $\left[A^{4}, G\right]$ and hence $i=0$ or 1 . But this additional information does not appear to shorten our argument.) By Corollary 2.8, $a$ cannot centralize both $\left[a_{i}, x\right]$ and $\left[a_{i}, y\right]$. Therefore, suppose without loss of generality that $a$ acts non-trivially on $\left\langle\left[a_{i}, x\right]\right\rangle$. Let $\left|\left[a_{i}, x\right]\right|=2^{t}$. So $t=n-i$, by Lemma 2.1 (iii). Since $a$ centralizes $\left[a_{i+1}, x\right], a$ must conjugate $\left[a_{i}, x\right]$ to $\left[a_{i}, x\right]^{\rho}$, where $\rho=2^{t-1}+1$, by Lemma 2.1 (iv). In the same way we see that $a$ either centralizes $\left[a_{i}, y\right]$ or conjugates it to $\left[a_{i}, y\right]^{\rho}$.

We have

$$
\left[a_{i}, y x\right]=\left[a_{i}, x\right]\left[a_{i}, y\right]\left[a_{i}, y, x\right] .
$$

Since $A$ centralizes $A_{i+1}\left[A_{i+1}, G\right]=A_{i+1}^{G}$, so also does $A^{G}$. Therefore, by Lemmas 2.3 and 2.6 we have

$$
\left[A_{i}, G, G\right] \leqslant A_{i+1}\left[A_{i+1}, G\right] \leqslant Z\left(A^{G}\right) .
$$

Therefore,

$$
a \text { centralizes }\left[a_{i}, y, x\right] \text {. }
$$

Suppose first that a centralizes $\left[a_{i}, y\right]$. Then it follows from (3.4) that

$$
a \text { centralizes }\left[a_{i}, x\right]^{-1}\left[a_{i}, y x\right] \text {. }
$$

Let $\left[a_{i}, y x\right]^{a}=\left[a_{i}, y x\right]^{\beta}$. From (3.7) we get

$$
\left[a_{i}, x\right]^{-\rho}\left[a_{i}, y x\right]^{\beta}=\left[a_{i}, x\right]^{-1}\left[a_{i}, y x\right] .
$$

But we can assume that $\langle[a, x]\rangle \cap\langle[a, y x]\rangle=1$, since otherwise Case 1 applies to $G=$ $\langle a, x, y x\rangle$. Therefore, $\rho \equiv 1 \bmod 2^{t}$, which is a contradiction.

Thus we may suppose that $\left[a_{i}, y\right]^{a}=\left[a_{i}, y\right]^{\rho}$. By Lemma 2.1,

$$
\left[A_{i}, G\right] /\left[A_{i+1}, G\right]
$$

is generated by elements of order 2 , all centralized by $A$ and therefore by $A^{G}$. So this quotient is elementary abelian. It follows from (3.5) that

$$
\left[A_{i}, G\right] \text { has class at most } 2 \text { and }\left[A_{i}, G\right]^{\prime} \text { is elementary abelian. }
$$


Since $\langle[a, x]\rangle \cap\langle[a, y]\rangle=1, a$ does not centralize $\left[a_{i}, x\right]\left[a_{i}, y\right]$. Therefore, by (3.4) and (3.6),

$$
a \text { does not centralize }\left[a_{i}, y x\right] \text {. }
$$

Clearly, $\left[A_{i}, G\right]$ has exponent $2^{t}$, by Lemmas 2.3 and 2.5. To complete the proof we distinguish two cases.

Suppose that $\left|\left[a_{i}, y x\right]\right|<2^{t}$. By Lemma 2.1 (iv), $t \geqslant 3$ and so $2^{t-1}=\rho-1 \geqslant 4$. Therefore, raising both sides of (3.4) to the power $\rho-1$, we get

$$
1=\left[a_{n-1}, x\right]\left[a_{n-1}, y\right]\left[a_{i}, y, x\right]^{\rho-1},
$$

using Lemma 2.1 (iii) and (3.8). Thus

$$
1 \neq\left[a_{n-1}, x\right]\left[a_{n-1}, y\right]=\left[a_{i}, y, x\right]^{\rho-1}=\left[\left[a_{i}, y\right]^{\rho-1}, x\right]=w,
$$

say, by (3.5). Hence $w=\left[a_{n-1}, y, x\right]$, again by Lemma 2.1 (iii). But by Lemmas 2.3 and 2.6,

$$
\left[A_{n-1}, G, G, G\right] \leqslant\left[A_{n}\left[A_{n}, G\right], G\right]=\left[A_{n}, G\right]=1 .
$$

Thus $w$ is a non-trivial element in $Z(G)$. Also $\left[a_{n-1}, x\right] \in\left[A_{i},\langle x\rangle, A\right] \leqslant K$ and similarly $\left[a_{n-1}, y\right] \in K$. Therefore, $w \in K$, contradicting $K_{G}=1$.

Finally, suppose that $\left|\left[a_{i}, y x\right]\right|=2^{t}$. Then, by $(3.9),\left[a_{i}, y x\right]^{a}=\left[a_{i}, y x\right]^{\rho}$ and hence, by (3.4) and (3.8), $\left[a_{i}, y, x\right]^{a}=\left[a_{i}, y, x\right]^{\rho}$. Thus from (3.6) we get $\left|\left[a_{i}, y, x\right]\right| \leqslant 2^{t-1}$. In the same way, interchanging $x$ and $y$, we may also assume that $\left|\left[a_{i}, x, y\right]\right| \leqslant 2^{t-1}$. Since $\left[A_{i}, G, G\right]$ is abelian (by (3.5)), it follows from Lemma 2.10 that $\left[A_{i}, G, G\right]$ has exponent $2^{t-1}$. Therefore, in centralizing $\left[A_{i}, G, G\right], a$ is in fact raising each element to its $\rho$ th power. Thus by Lemma 2.9 and $(3.9), a$ conjugates each element of $\left[A_{i}, G\right]$ to its $\rho$ th power.

Putting $L=\left[A_{i}, G\right]$, we now have $L^{\rho-1} \leqslant[L, A] \leqslant L^{\rho-1}$. So $[L, A]=L^{\rho-1} \triangleleft G$. But $1 \neq[L, A] \leqslant K$, contradicting $K_{G}=1$. This completes the proof of Theorem 1.2 (i).

\section{Proof of Theorem 1.2 (ii)}

Here $A=\langle a\rangle$ is a cyclic permutable subgroup of a finite 2-group $G$ and we must show that $[A, G, A]=\{[u, a] \mid u \in[A, G]\}$. We argue by induction on $|A|$. If $|A| \leqslant 4$, then $|[a, g]| \leqslant 4$ for all $g \in G$, by Lemma 2.1 (iii). Thus $a$ centralizes all $[a, g]$, by Lemma 2.1 (iv), so $[A, G, A]=1$ and the theorem is true. Therefore, suppose that $|A| \geqslant 8$ and assume the usual induction hypothesis. Since $A^{2}$ is permutable in $G$ by Lemma 2.3 , we deduce that

$$
\left[A^{2}, G, A^{2}\right]=\left\{\left[v, a^{2}\right] \mid v \in\left[A^{2}, G\right]\right\}=K,
$$

say. By Theorem $1.2(\mathrm{i}), K \triangleleft G$. By Lemma 2.1 (ii), a normalizes each cyclic subgroup $\left\langle\left[v, a^{2}\right]\right\rangle$ and so $A$ normalizes every subgroup of $K$. Then $A^{G}(\geqslant[A, G])$ does the same and hence $K$ has all its subgroups normal. Suppose that $K$ is not abelian, so it is the direct product of a quaternion group of order 8 and an elementary abelian 2-group (as we saw in the proof of Theorem 1.3). But then the commutators $\left[v, a^{2}\right]$ all have order at 
most 4 and are centralized by $a$, by Lemma 2.1 (iv). Thus $A$ centralizes $K$ and therefore $K \leqslant Z\left(A^{G}\right)$, contradicting the fact that $K$ is not abelian. So

$$
K \text { is abelian. }
$$

By analogy with (2.1) in the proof of Theorem 1.3, we see that

$$
[K,[A, G]]=1 .
$$

Let $v \in\left[A^{2}, G\right]$. By Lemma 2.1 (ii), there are elements $g_{1}, \ldots, g_{n}$ in $G$ such that $v=\left[a^{2}, g_{1}\right] \ldots\left[a^{2}, g_{n}\right]$. Therefore, by $(4.1)$,

$$
\left[v, a^{2}\right]=\left[\left[a^{2}, g_{1}\right] \ldots\left[a^{2}, g_{n}\right], a^{2}\right]=\left[a^{2}, g_{1}, a^{2}\right] \ldots\left[a^{2}, g_{n}, a^{2}\right] .
$$

But $\left\langle\left[a^{2}, g_{i}, a^{2}\right]\right\rangle=\left\langle\left[\left[a^{2}, g_{i}\right]^{2}, a\right]\right\rangle$, by Lemma 2.1 applied to the product $\left\langle\left[a^{2}, g_{i}\right]\right\rangle\langle a\rangle$, in which both factors are permutable subgroups. Therefore, with $M=\left\langle\left[a^{2}, g_{i}\right]^{2}\right\rangle\langle a\rangle$, we have $M^{\prime}=\left\langle\left[a^{2}, g_{i}, a^{2}\right]\right\rangle$. Again by Lemma 2.1, each element of $M^{\prime}$ has the form $[x, a]$, with $x \in\left\langle\left[a^{2}, g_{i}\right]^{2}\right\rangle \leqslant\left[A^{4}, G\right]$. Thus $\left[a^{2}, g_{i}, a^{2}\right]=\left[x_{i}, a\right]$ for some element $x_{i} \in\left[A^{4}, G\right]$. Therefore,

$$
\left[v, a^{2}\right]=\left[x_{1}, a\right] \ldots\left[x_{n}, a\right]=\left[x_{1} \ldots x_{n}, a\right]
$$

by (4.1). It follows that every element of $K$, as an element of $[A, G, A]$, has the form that we are trying to establish. Since $K \triangleleft G$, there is a central series of $G$ passing through $K$. Then using the identity $\left[u_{1} u_{2}, a\right]=\left[u_{1}, a\right]^{u_{2}}\left[u_{2}, a\right]$, a simple induction allows us to assume that

$$
K=\left[A^{2}, G, A^{2}\right]=1 .
$$

Using Lemma 2.1 again, we see that $\left[A, G, A^{2}\right]$ is generated by elements of the form

$$
\left[\left[a, h_{1}\right] \ldots\left[a, h_{r}\right], a^{2}\right]=\prod_{i=1}^{r}\left[a, h_{i}, a^{2}\right]^{c_{i}},
$$

for suitable elements $h_{i}$ in $G$ and $c_{i}=\left[a, h_{i+1}\right] \ldots\left[a, h_{r}\right]$. By (4.2), $a$ induces an automorphism of order at most 2 in any subgroup of the form $\left\langle\left[a^{2}, g\right]\right\rangle$. So by Lemma 2.1 (iv), $a$ centralizes $\left\langle\left[a^{2}, g\right]^{2}\right\rangle=\left\langle\left[a^{4}, g\right]\right\rangle$. Therefore, $A$ centralizes $\left[A^{4}, G\right]$ and hence so also does $A^{G}=A[A, G]$. But again by Lemma 2.1 (iv), we have

$$
\left[a, g, a^{2}\right] \in\left\langle[a, g]^{8}\right\rangle \leqslant\left[A^{4}, G\right]
$$

and therefore each $\left[a, h_{i}, a^{2}\right]$ commutes with $c_{i}$ in (4.3). Thus $\left[A, G, A^{2}\right]$ is generated by the elements $\left[a, g, a^{2}\right], g \in G$.

Choose $g \in G$ and let $L=\left\langle[a, g]^{2}\right\rangle\langle a\rangle$, so that $L^{\prime}=\left\langle\left[[a, g]^{2}, a\right]\right\rangle=\left\langle\left[a, g, a^{2}\right]\right\rangle$. Each element of $L^{\prime}$ has the form $[y, a]$, where $y \in\left\langle[a, g]^{2}\right\rangle=\left\langle\left[a^{2}, g\right]\right\rangle \leqslant\left[A^{2}, G\right]$. Therefore, $\left[a, g, a^{2}\right]=[y, a]$, for such an element $y$ in $\left[A^{2}, G\right]$. It follows that $\left[A, G, A^{2}\right]$ is generated by elements of the form $[y, a]$. But with $y_{1}, y_{2} \in\left[A^{2}, G\right]$, we have $\left[y_{1}, a\right]\left[y_{2}, a\right]=\left[y_{1} y_{2}, a\right]$, by (4.2). Thus $\left[A, G, A^{2}\right] \subseteq\left\{[y, a] \mid y \in\left[A^{2}, G\right]\right\}$. In particular, $\left[A, G, A^{2}\right] \leqslant\left[A^{2}, G, A\right]$. Conversely, $\left[A, G, A^{2}\right]=[A, G, A]^{2}$, by Lemma 2.4 (replacing $G$ there by $A^{G}=A[A, G]$ ). 
Therefore, $\left[A, G, A^{2}\right] \triangleleft G$, by Theorem $1.2(\mathrm{i})$, and it follows from the Three Subgroup Lemma (see $[8,5.1 .10])$ that $\left[A^{2}, G, A\right] \leqslant\left[A, G, A^{2}\right]$. Thus

$$
\left[A, G, A^{2}\right]=\left[A^{2}, G, A\right]=\left\{[y, a] \mid y \in\left[A^{2}, G\right]\right\} \triangleleft G .
$$

Just as we were able to assume above that $K=1$, so now from (4.4) we may suppose that

$$
\left[A, G, A^{2}\right]=\left[A^{2}, G, A\right]=1 .
$$

By Lemma 2.1 we have $[A, G, A] \leqslant\left[A^{4}, G\right]$, and $\left[A^{2}, G,[A, G]\right]=1$, by (4.5). Therefore,

$$
[A, G, A,[A, G]]=1 \text {. }
$$

Each element of $[A, G]$ has the form $\left[a, y_{1}\right] \ldots\left[a, y_{m}\right], y_{i} \in G$, by Corollary 2.2. Thus $[A, G, A]$ is generated by elements of the form

$$
\left[\left[a, y_{1}\right] \ldots\left[a, y_{m}\right], a\right]=\left[a, y_{1}, a\right] \ldots\left[a, y_{m}, a\right],
$$

by (4.6), i.e. $[A, G, A]$ is generated by elements $[a, g, a], g \in G$. But, by (4.7), products of such elements have the form $[u, a], u \in[A, G]$. This completes the proof of Theorem 1.2 (ii).

\section{References}

1. R. BAER, Situation der Untergruppen und Struktur der Gruppen, Sitz. Ber. Heidelberg Akad. 2 (1933), 12-17.

2. C. D. H. CoOPER, Power automorphisms of a group, Math. Z. 107 (1968), 335-356.

3. J. Cossey and S. E. Stonehewer, Cyclic permutable subgroups of finite groups, $J$. Aust. Math. Soc. 71 (2001), 169-176.

4. J. Cossey And S. E. Stonehewer, The embedding of a cyclic permutable subgroup in a finite group, Illinois J. Math., in press.

5. R. Dedekind, Über Gruppen, deren sämtliche Teiler Normalteiler sind, Math. Annln 48 (1897), 548-561.

6. B. HupPERT, Über das Produkt von paarweise vertauschbaren zyklischen Gruppen, Math. Z. 58 (1953), 243-264.

7. O. Ore, On the application of structure theory to groups, Bull. Am. Math. Soc. 44 (1938), 801-806.

8. D. J. S. Robinson, A course in the theory of groups, 2nd edn, Graduate Texts in Mathematics, vol. 80 (Springer, 1996).

9. R. Schmidt, Subgroup lattices of groups, Expositions in Mathematics, vol. 14 (de Gruyter, Berlin, 1994). 